\title{
A quantitative ultrastructural study of juxtaglomerular arterioles in IDDM patients with micro-and normoalbuminuria
}

\author{
R. Østerby ${ }^{1}$, H-J. Bangstad ${ }^{2}$, G. Nyberg ${ }^{3}$, J. D. Walker ${ }^{4}$, GC. Viberti ${ }^{4}$ \\ ${ }^{1}$ Electron Microscopy Laboratory, Institute for Experimental Clinical Research, Medical Department M (Diabetes and \\ Endocrinology) and University Institute of Pathology, Århus Kommunehospital, Århus, Denmark \\ ${ }^{2}$ Aker Diabetes Research Centre, Aker University Hospital, Oslo, Norway \\ ${ }^{3}$ Transplant Unit, Sahlgrenska University Hospital, Göteborg, Sweden \\ ${ }^{4}$ Unit for Metabolic Medicine, United Medical and Dental Schools, Guy's Hospital, London, UK
}

Summary Hyalinization of juxtaglomerular arterioles is prominent in advanced diabetic nephropathy and may have important functional consequences. We studied the early stages of diabetic renal disease using kidney biopsy material from insulin-dependent diabetic patients, 8 with normal albumin excretion rate $(<15 \mu \mathrm{g} / \mathrm{min})$ and 16 with microalbuminuria (15-200 $\mu \mathrm{g} / \mathrm{min})$. Ten living non-diabetic kidney donors served as a control group. Median duration of diabetes was 9.5 years (range 5-31) in patients with normoalbuminuria, and 12 years (7-22) in patients with microalbuminuria $(p=0.27)$. The tissue was sectioned systematically, $1-\mu \mathrm{m}$ thick sections for light microscopy at $10-\mu \mathrm{m}$ intervals, and thin sections for electron microscopy taken at $60-\mu \mathrm{m}$ intervals. The arterioles were identified as afferent or efferent, and total profiles were photographed (magnification $7500 \times$ ), providing a systematic independent sample for measurements using standard stereological methods. Patients with microalbuminuria had significantly increased arteriole parameters compared with the control group: for afferent and efferent arterioles the volume fraction of matrix/media, means and (coefficient of variation, CV), was $0.47(0.16)$ vs 0.33 (0.19) $(p=0.0009)$, and $0.62(0.14)$ vs $0.45(0.23)$ $(p=0.0004)$ and matrix-T, expressing amount of matrix per unit arteriolar surface, $2.38(0.38) \mu \mathrm{m}$ vs 1.44 (0.30) $\mu \mathrm{m}(p=0.004)$, and $1.62(0.28) \mu \mathrm{m}$ vs 1.03 (0.34) $(p=0.0009)$. Patients with normoalbuminuria showed no significant differences from the control group, and had lower values than microalbuminuric patients for all parameters except the afferent matrix- $T$. In the normoalbuminuric group a correlation was found between parameters for afferent arterioles and those for glomerular structure. In conclusion there is arteriolar accumulation of extracellular material in the early phase of diabetic nephropathy, concomitant with early glomerulopathy. [Diabetologia (1995) 38: 1320-1327]

Key words Diabetic nephropathy, diabetic glomerulopathy, arteriolar hyalinosis, juxtaglomerular arterioles, extracellular matrix, microalbuminuria, stereology.
The juxtaglomerular arterioles play an important role in the regulation of glomerular function [1]. In insu-

Received: 13 March 1995 and in revised form: 4 May 1995

Corresponding author: Dr. R. Østerby, Electron Microscopy Laboratory, Building 03, Århus Kommunehospital, DK-8000 Århus C, Denmark

Abbreviations: IDDM, Insulin-dependent diabetes mellitus; GFR, glomerular filtration rate; AER, albumin excretion rate; matrix-T, matrix thickness; ND, non-diabetic subjects; $\mathrm{D}_{\mathrm{NA}}$, IDDM patients with normal albumin excretion rate; $\mathrm{D}_{\mathrm{MI}}$, IDDM patients with microalbuminuria; RPF, renal plasma flow; $\mathrm{CV}$, coefficient of variation. lin-dependent diabetic (IDDM) patients glomerular function is disturbed by raised glomerular filtration rate (GFR), renal plasma flow (RPF) and filtration fraction in the early phase of the disease [2]. Increased intraglomerular pressure has been observed in experimental diabetes $[3,4]$. This has led to postulate a role for elevated glomerular pressure in the development of glomerulopathy. It is not possible to directly measure glomerular pressure in human subjects, but the measurable functional changes indicate a disturbed balance of arteriolar function.

Diabetic patients with advanced nephropathy, i.e. reduced and decreasing GFR, present striking renal 
arteriolar lesions in addition to the full-blown glomerulopathy. Arteriolar hyalinosis has been observed in various stages of the disease, and semiquantitative examinations indicate that the severity parallels glomerular pathology, as well as the clinical state [5-9].

The present study was performed in order to obtain quantitative data on arteriolar structure in the earliest phase of diabetic nephropathy, characterized by microalbuminuria, compared with findings in diabetic patients with normal urinary albumin excretion and with control subjects. Patients with microalbuminuria are at high risk of progressing to more advanced renal disease over time [10]. We have previously obtained data on the glomerular structures in these patients, demonstrating definite differences with groups of diabetic patients who were normoalbuminuric [11].

\section{Subjects and methods}

Patients. All patients in the present series have been included in previous publications on glomerular ultrastructure $[11,12]$. Those patients who had elevated blood pressure according to World Health Organization criteria at the time of biopsy were excluded from the total series: one with normal albumin excretion [11], one with microalbuminuria [12], and six initially microalbuminuric patients who did not have persistent microalbuminuria after a follow-up period of 2-3 years [13].

The IDDM patients participated in a study of the natural history of diabetic nephropathy in IDDM [11], or in a clinical intervention trial of intensified treatment of young microalbuminuric patients [12]. From the latter follow-up study only the baseline biopsies taken at entry into the trial have been studied. The grouping of the diabetic patients was based on the albumin excretion rate (AER), which was measured in three timed overnight sterile urine samples collected during the year preceding the biopsy. The lower limit for microalbuminuria was set to $15 \mu \mathrm{g} / \mathrm{min}$, in at least two out of three consecutive overnight samples. The non-diabetic control subjects were living donors for renal transplantation, the biopsy being taken at the time of operation. A thorough clinical investigation was performed prior to donation, to ensure normal renal function, and to exclude the presence of diabetes.

Table 1 shows clinical data in the groups of kidney donors (ND), IDDM patients with normoalbuminuria, $\mathrm{D}_{\mathrm{NA}}$, and IDDM patients with microalbuminuria, $\mathrm{D}_{\mathrm{MI}}$. The age of the $D_{M I}$ patients was significantly lower than in the other groups $(p=0.02)$. The difference in duration of diabetes was not statistically significant $(p=0.27)$. Glomerular filtration rate was measured in diabetic patients by ${ }^{51} \mathrm{CrEDTA}$ clearance after a single intravenous injection or by inulin clearance during steady-state water diuresis. Blood pressure was taken to the nearest $2 \mathrm{~mm} \mathrm{Hg}$, using a standard mercury sphygmomanometer, with the patients sitting after 10 min rest. The mean values of several measurements are given. Diastolic blood pressure was significantly. increased in the $\mathrm{D}_{\mathrm{MI}}$ group vs $\mathrm{D}_{\mathrm{NA}}(p=0.01)$, as was also the average $\mathrm{HbA}_{1 \mathrm{c}}$ during the year preceding the biopsy $(p=0.0009)$.

Renal biopsies. In the IDDM patients needle biopsies were taken using a Tru-cut or a Biopty 18-gauge needle under ultra-
Table 1. Clinical data in the series

\begin{tabular}{|c|c|c|c|}
\hline & ND & $\mathrm{D}_{\mathrm{NA}}$ & $\mathrm{D}_{\mathrm{MI}}$ \\
\hline$n$ & 9 & 8 & 16 \\
\hline Age (years) & $\begin{array}{l}28 \\
(20-34)\end{array}$ & $\begin{array}{l}32 \\
(19-39)\end{array}$ & $\begin{array}{l}19 \\
(17-43)\end{array}$ \\
\hline $\begin{array}{l}\text { Sex (Female, } \\
\text { Male) }\end{array}$ & $5 \mathrm{~F} / 4 \mathrm{M}$ & $4 \mathrm{~F} / 4 \mathrm{M}$ & $7 \mathrm{~F} / 9 \mathrm{M}$ \\
\hline $\begin{array}{l}\text { Diabetes duration, } \\
\text { years }\end{array}$ & & $\begin{array}{l}9.5 \\
(5-31)\end{array}$ & $\begin{array}{l}12 \\
(7-22)\end{array}$ \\
\hline $\mathrm{HbA}_{\mathrm{Ic}}$ & & $\begin{array}{l}7.2 \\
(6.1-9.7)\end{array}$ & $\begin{array}{l}9.3 \\
(7.9-12.6)\end{array}$ \\
\hline $\mathrm{AER}, \mu \mathrm{g} / \mathrm{min}$ & $\begin{array}{l}\text { dipstick } \\
\text { negative }\end{array}$ & $\begin{array}{l}4.9 \\
(2.1-7.2)\end{array}$ & $\begin{array}{l}36.5 \\
(16-224)\end{array}$ \\
\hline $\begin{array}{l}\mathrm{GFR}, \mathrm{ml} \cdot \min ^{-1} . \\
1.73 \mathrm{~m}^{-2}\end{array}$ & not done & $\begin{array}{l}124 \\
(96-176)\end{array}$ & $\begin{array}{l}133 \\
(101-193)\end{array}$ \\
\hline $\begin{array}{l}\text { Systolic blood } \\
\text { pressure, mm Hg }\end{array}$ & $\begin{array}{l}114 \\
(102-138)\end{array}$ & $\begin{array}{l}121 \\
(106-127)\end{array}$ & $\begin{array}{l}127 \\
(90-145)\end{array}$ \\
\hline $\begin{array}{l}\text { Diastolic blood } \\
\text { pressure, } \mathrm{mm} \mathrm{Hg}\end{array}$ & $\begin{array}{l}70 \\
(64-92)\end{array}$ & $\begin{array}{l}72 \\
(51-77)\end{array}$ & $\begin{array}{l}78 \\
(52-90)\end{array}$ \\
\hline
\end{tabular}

Values are median (range)

sound guidance. The whole tissue core was mailed in a fixative ( $2 \%$ glutaraldehyde in buffer) to the laboratory in Arhus, where dehydration and embedding into Vestopal were performed. From the control subjects a biopsy was taken during transplantation, either as a needle or a wedge biopsy. The embedding medium was Vestopal in two cases and epon in the remainder.

Sectioning and sampling for electron microscopy. The blocks of kidney tissue processed for electron microscopy were sectioned systematically; semithin sections were picked up for light microscopy at $10-\mu \mathrm{m}$ intervals, and large thin sections for electron microscopy, covering the full extent of the block, at 60- $\mu \mathrm{m}$ intervals. The first section of tissue in each block was numbered 0 , and the thin sections taken at predetermined levels at $60 \mu \mathrm{m}$ or multiples thereof, from section number 0 . This protocol was originally designed to obtain a systematic, independent sample of glomerular profiles [11]. The arteriolar profiles were localized by light microscopy, and identified as afferent or efferent by following their course in sequential sections. From the thin sections an independent, systematic sample was obtained, photographing all arteriolar profiles present in the sections. Arteriolar profiles were photographed to their full extent, producing photomontages when necessary. The final magnification on prints was $7400 \times$, calculated for each biopsy by simultaneous photographing of a calibration grid with 2160 lines per $\mathrm{mm}$. The number of arteriolar profiles obtained in each case is given in Table 2. The minimal accepted number of profiles per case was 5 . One control case was omitted for the calculation of afferent arterioles, and one for the efferent arterioles, due to too few available profiles. A profile may represent highly varying areas, depending on whether a transverse or longitudinal section was cut, and Table 2 also gives the areas sampled, and a calculated value corresponding to the length of arteriolar wall. The latter figure is included due to the easier perception of one-dimensional rather than two-dimensional measures, and it is calculated as area of media/media-T.

Morphometry. The applied descriptor of arteriolar structure was the volume fraction of extracellular material, matrix, using the media as reference space. Media is the layer extend- 
Table 2. Material studied by electron microscopy in individual biopsies

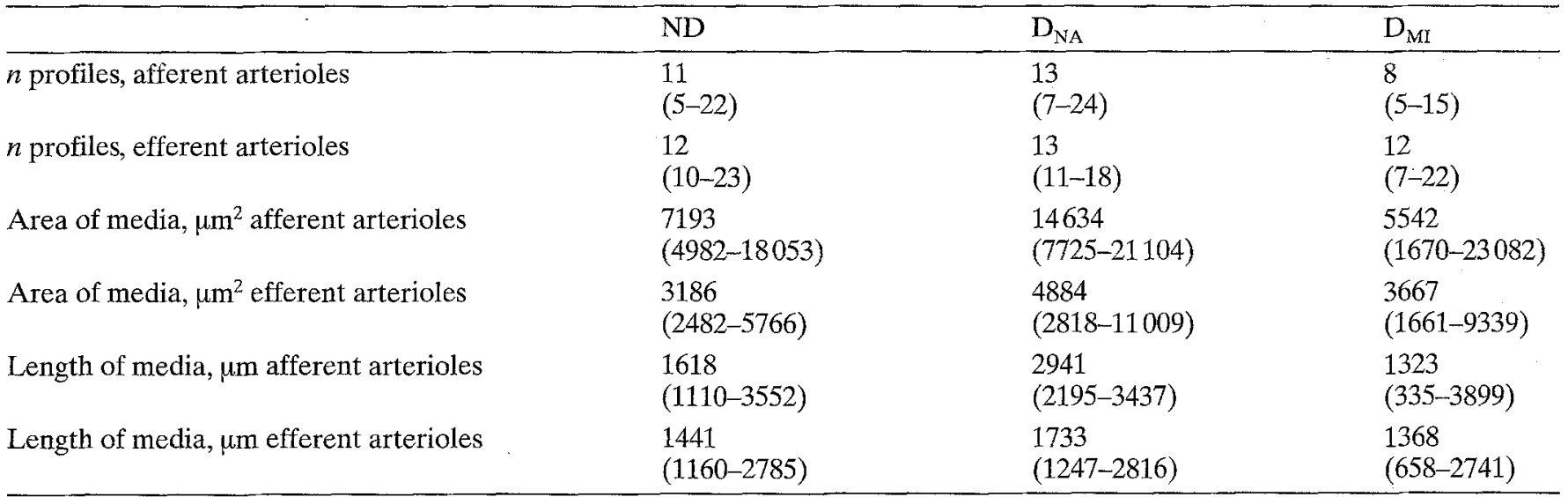

Values are median (range)

ing from the external aspect of endothelial cytoplasm to the surrounding interstitial tissue (Fig. 1). It consists of the myomedial cells and surrounding matrix. The estimate of volume fraction was obtained by simple point counting, the distance between points corresponding to $3 \mu \mathrm{m}$. Further, the fraction of electron-dense areas per matrix was estimated, since such darkly-stained, localized areas are typical of arteriolar hyalinosis. Another estimate of matrix was calculated, the matrix-T, which is the arbitrary matrix thickness, i. e. the thickness of matrix if spread in an even layer on the arteriolar circumference $[11,12]$. It is calculated from volume/surface ratio, using the estimated densities, volume fraction of matrix, $\mathrm{V}_{\mathrm{V}}$ (matrix/media) and surface density, $S_{V}$ (arteriolar circumference/media). Surface density was estimated by standard stereology technique, counting intersections between test lines and the trace of arteriolar circumference.

\section{Statistical analysis}

Differences among groups were tested by Kruskall Wallis' analysis of variance, and comparisons between pairs of groups using the Mann-Whitney test and Bonferroni correction. Correlations among variables were tested by least square regression. Actual $p$-values are given. Differences with $p$ greater than 0.05 are considered not significant (NS). The group results are given as the mean value and the $C V$, $\mathrm{CV}=\mathrm{SD} / \mathrm{mean}$.

\section{Results}

Results from individual biopsies are plotted in Figures 2-5, separately for afferent and efferent arterioles. The volume fraction, $V_{V}$ (matrix/media) in the three groups of control subjects, normo-and microalbuminuric diabetic patients, respectively was, mean and (CV) for the afferent arterioles (Fig. 2): 0.33 $(0.19), 0.36(0.27)$ and $0.47(0.16)$. The microalbuminuric patients had a significantly increased value compared with control subjects $(p=0.0009)$ and normoalbuminuric patients $(p=0.012)$. For the efferent arterioles the volume fractions in the same groups were
(Fig. 3): $0.45(0.23), 0.35(0.24)$ and $0.62(0.14)$. The microalbuminuric patients had statistically significantly elevated values compared to control subjects $(p=0.0015)$ and to normoalbuminuric patients $(p=0.0001)$.

Matrix-T in afferent arterioles (Fig. 4) was $1.44 \mu \mathrm{m}$ $(0.30), 1.97 \mu \mathrm{m}(0.37)$ and $2.38 \mu \mathrm{m}(0.38)$, i. e. a significantly thicker matrix in microalbuminuric patients compared with control subjects $(p=0.004)$, but not significantly different compared with the normoalbuminuric group. In efferent arterioles (Fig. 5) matrix-T was $1.03 \mu \mathrm{m}(0.28), 1.03 \mu \mathrm{m}(0.34)$ and $1.62 \mu \mathrm{m}$ $(0.28)$. Thus, matrix thickness was increased in microalbuminuric patients compared with normoalbuminuic patients $(p=0.004)$ and controls $(p=0.003)$. Media thickness did not show any differences among the groups, for either afferent or efferent arterioles. Total wall thickness, i. e. including the endothelial cells, was increased in efferent arterioles in the $D_{\mathrm{NA}}$ group, compared to control subjects $(p=0.0014)$ and to the $\mathrm{D}_{\mathrm{MI}}$ group $(p=0.025)$.

In the $\mathrm{D}_{\mathrm{M}}$ group there was a correlation between matrix- $T$ in afferent and efferent arterioles $(\mathrm{r}=0.55$, $p=0.029$ ), but no other significant correlation was found pertaining to afferent and efferent arterioles within biopsies.

The volume of electron-dense areas as fraction of total matrix failed to show significant differences among the groups, although for the afferent arterioles the value in the control group $(-6 \%)$ was lower than the approximately $8-10 \%$ measured in diabetic patients.

Significant correlations were obtained in the $\mathrm{D}_{\mathrm{NA}}$ group between structural parameters pertaining to the afferent arterioles and those characterizing the glomerulopathy (Table 3, Fig.6), but not to those of the efferent arterioles, or with the duration of diabetes. In the patients with microalbuminuria no clear correlation between glomerulopathy and arteriolar lesions was found. 


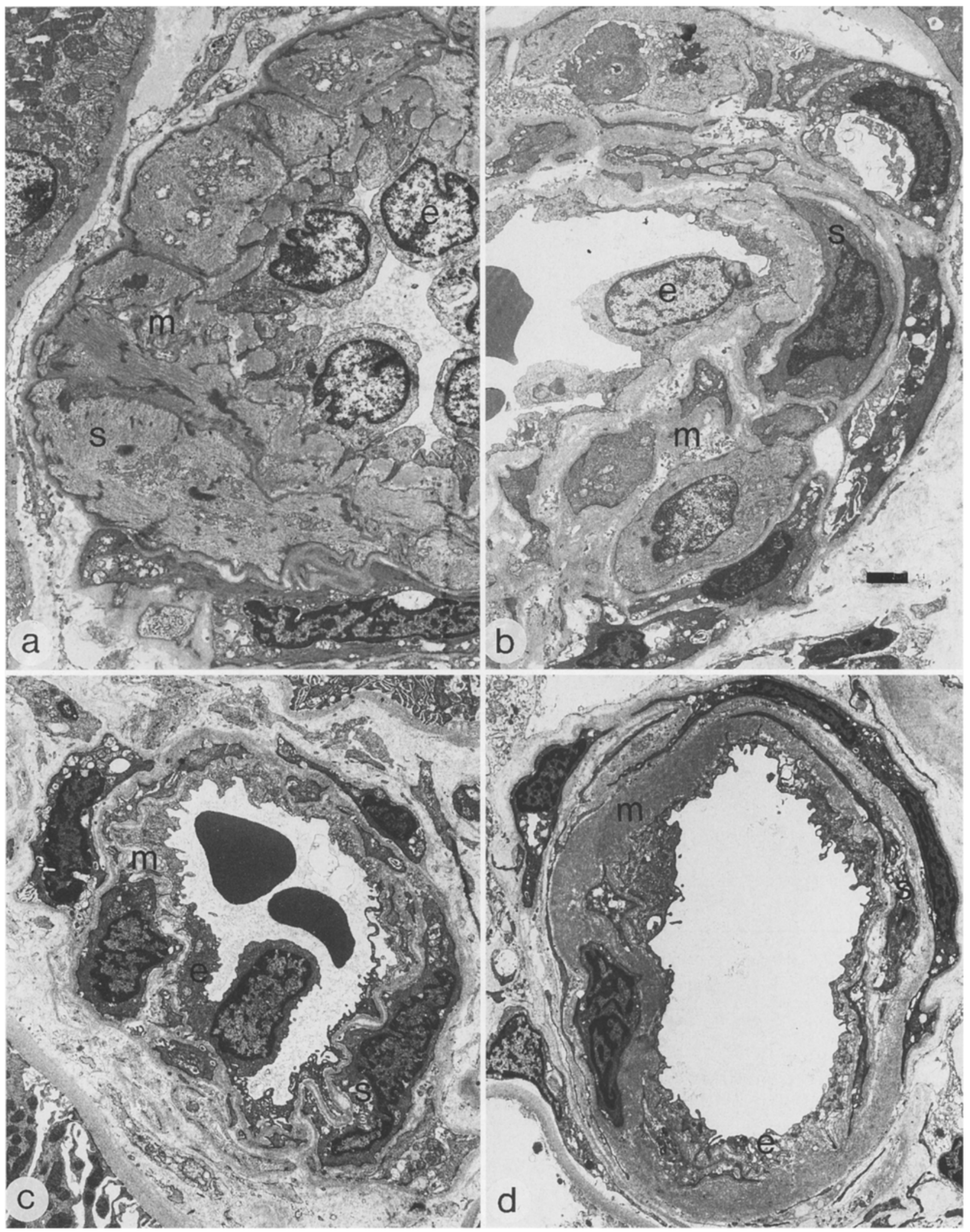

Fig. 1a-d Sections of arteriolar profiles. $e$, Endothelium; $s$, smooth muscle cell; $m$, matrix, surrounding the muscle cells. Muscle cells + matrix constitute the media. The bar (section b) indicates $1 \mu \mathrm{m}$. a Afferent arteriole in which the smooth muscle cells constitute the major part of media, the matrix appearing only as a layer beneath the endothelium and as thin sheets surrounding the smooth muscle cells. b An afferent arteriole in which the distances between smooth muscle cells are increased due to augmentation of matrix. c, d Cross-sections of efferent arterioles. A larger fraction of matrix per media is present in section $\mathbf{d}$ compared with $\mathbf{c}$ 


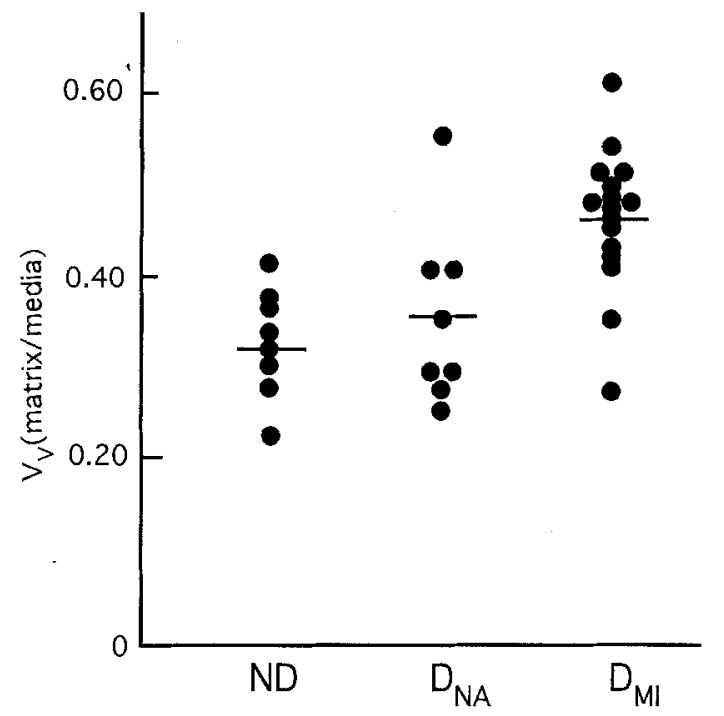

Fig. 2. Matrix in afferent arterioles expressed as volume fraction of media in the groups of non-diabetic living kidney donors (ND), in IDDM patients with normal albumin excretion $\left(D_{N A}\right)$ and in IDDM patients with microalbuminuria $\left(D_{M I}\right)$

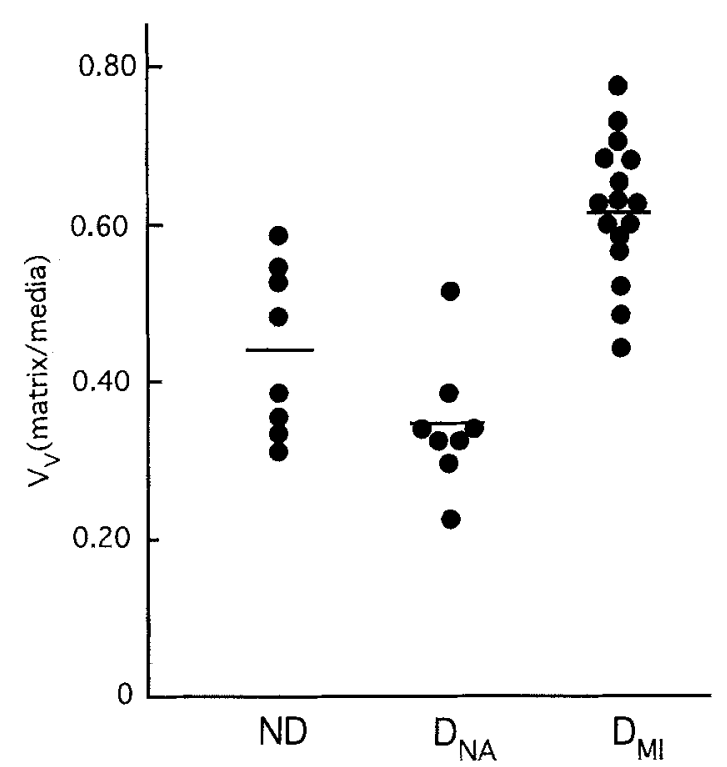

Fig. 3. Volume fraction of matrix per media in efferent arterioles

No correlations were seen within groups between arteriolar structure and systemic blood pressure or AER.

\section{Discussion}

The results of the present study show that structural lesions are present in the juxtaglomerular afferent and efferent arterioles at the stage of microalbuminuria. Diabetic patients with normoalbuminuria had some changes in afferent arterioles implying that as far as can be inferred from this cross-sectional

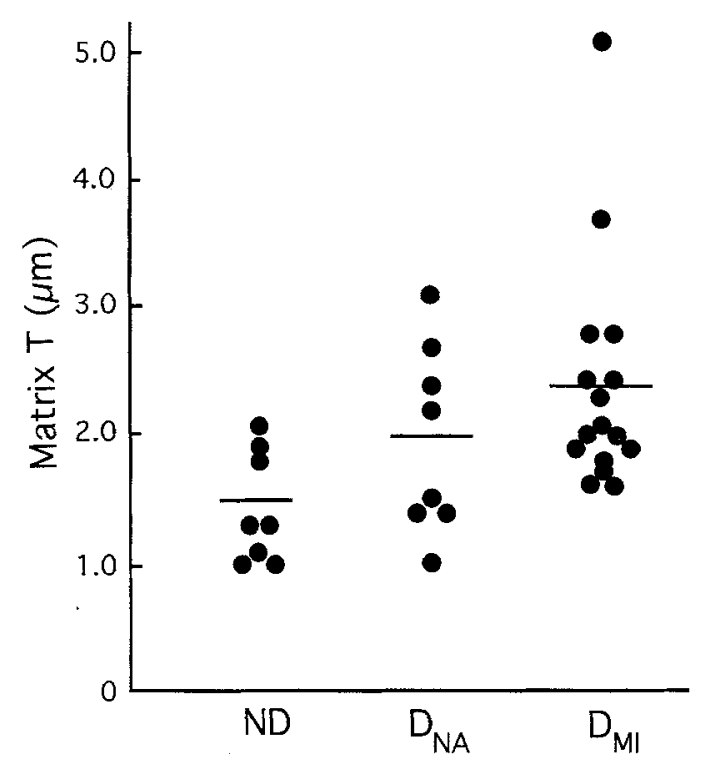

Fig. 4. Estimated matrix arbitrary thickness in afferent arterioles; i.e. the average height of matrix carried per unit arteriolar circumference

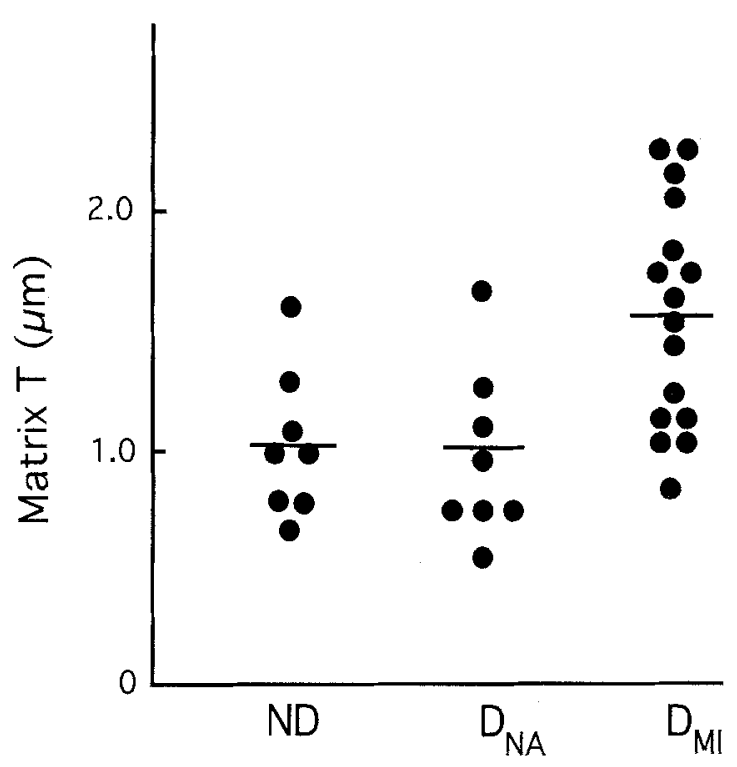

Fig.5. Estimated matrix arbitrary thickness in efferent arterioles

study - this may be the first site to be affected, followed later by changes in the efferent arterioles. The normoalbuminuric patients who showed abnormalities in afferent arterioles also had signs of glomerulopathy, but the changes were very moderate, in both glomerular and in arteriolar structure. These observations are at variance with a recent publication, reporting extremely advanced lesions in some patients with normal albumin excretion [14]. We also found moderate glomerular changes in the low microalbuminuria range $[12,13]$.

The increase in matrix volume fraction in the IDDM patients with microalbuminuria could be a 


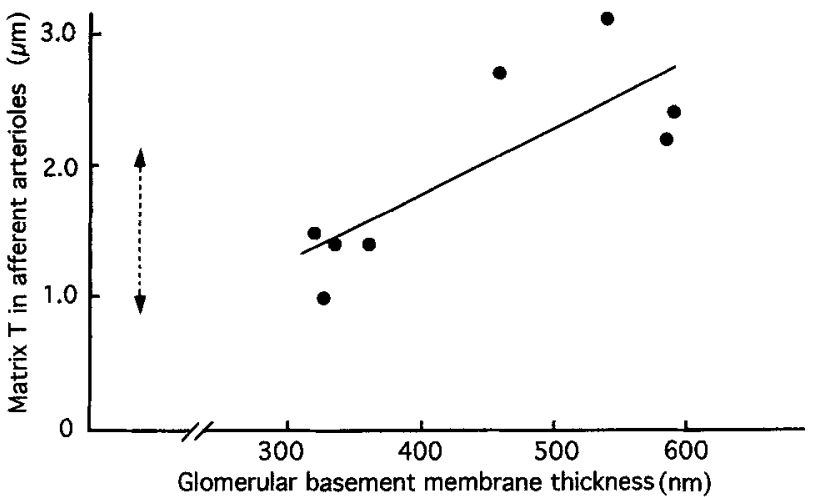

Fig. 6. Correlation between structure in afferent arterioles and in glomeruli in the group of IDDM patients with normal albumin excretion. The arteriolar parameter on the Y-axis is matrix thickness, $\mathrm{T}$, and the $\mathrm{X}$-axis shows glomerular basement thickness. The stippled line indicates the range in the ND group. $r=0.82 p=0.012$

result solely of loss of myomedial cells, yet, the increased matrix-thickness indicates that accumulation of matrix has taken place. The change observed in arteriole wall composition is analogous to the initial changes in the mesangial regions, also characterized by augmentation of the extracellular matrix $[15,16]$. In the $D_{\mathrm{NA}}$ group an unexpected decrease in $\mathrm{V}_{\mathrm{V}}$ (matrix/media) in efferent arterioles was seen, synonymous with an increase in the volume fraction of smooth muscle cells. Since matrix-T at the same time was similar to that in the control group, and media thickness tended to be increased, the results indicate that efferent smooth muscle cell hypertrophy may be present in this group. However, these differences were only borderline significant, and the question should be pursued in further studies before firm conclusions are drawn.

The variables measured had a fairly large withingroups variation with a CV of about $20-30 \%$. Calculated thickness requires a uniform orientation distribution between vessel axis and the plane of sectioning. Based on the serial sectioning method employed, and following vessel profiles in sequential sections, the arterioles could be identified irrespective of the angle of sectioning at the level of the thin sections. Whether the present frequent sampling was sufficient to satisfy the requirement of orientation distribution cannot be established. Another condition which necessitated extensive sampling was the patchy occurrence of matrix accumulation, and also the fact that some young kidney donors, irrespective of normal renal function and normal clinical blood pressure, showed localized hyalinization of arterioles. The variation among individual arteriolar profiles within one biopsy was often large. It appeared that structural lesions were often most pronounced in close proximity to the glomerulus. Since the distance to the glomerulus could not always be deter-
Table 3. Correlations between structure in afferent arterioles and in glomeruli in $\mathrm{D}_{\mathrm{NA}}(n=8)$

\begin{tabular}{llllll}
\hline & \multicolumn{3}{l}{ Afferent arterioles } & \\
\cline { 2 - 3 } & \multicolumn{2}{l}{$\mathrm{V}_{\mathrm{V}}$ (matrix/media) } & & \multicolumn{2}{l}{ matrix $\mathrm{T}$} \\
\cline { 2 - 3 } \cline { 5 - 6 } & $r$ & $p$ & & $r$ & $p$ \\
\hline Glomerular BMT (nm) & 0.74 & 0.036 & & 0.82 & 0.012 \\
Index (glomerulopathy) & 0.72 & 0.04 & & 0.85 & 0.007 \\
\hline
\end{tabular}

BMT, Basement membrane thickness; index (glomerulopathy), BMT/10+ $\mathrm{V}_{\mathrm{v}}$ (matrix/glomerulus) $\cdot 100$

mined, it was decided to include any level, from the glomerular vascular pole to a larger artery for the afferent vessels, and from the pole to the level of interstitial capillaries for the efferent arterioles. An explanation for the variation between different locations within biopsies is not available. The degree of arteriolar injury may contribute, in the long run, to the heterogeneity of glomerulopathy which is seen in kidneys with advanced disease, and may be important in explaining the mechanism leading to glomerular occlusion. There is at present no other good explanation of why some glomeruli become totally occluded, while others are preserved. In the early phases of diabetic glomerulopathy the lesions appear more uniformly distributed among nephrons [12].

It seems reasonable to consider the arteriolar accumulation of matrix as another aspect of the abnormal basement membrane metabolism in diabetes, which in glomeruli is expressed as the basement membrane thickening and accumulation of mesangial matrix. In vitro experiments have shown increased matrix production when endothelial [17], or mesangial cells [18-21], are grown in high glucose medium. In agreement with observations in other studies [22-24] the group of patients with microalbuminuria had a higher $\mathrm{HbA}_{1 \mathrm{c}}$ level than the patients with normal AER. Long-term poor metabolic control definitely appears to be a very important factor in the development of the structural lesions [13, 25].

The changes in arteriolar wall structure as observed in this series - whether initiated by metabolic aberrations, or influenced by hydrostatic forces - are likely to have a marked impact on arteriolar function. The larger proportion of the wall being matrix, and less smooth muscle cells, the arterioles would be less capable of responding to immediate changes in systemic pressure, or to messages from the juxtaglomerular apparatus, endothelium or nerve impulses. Glomerular capillaries downstream the afferent arterioles would be less protected from intermittent increases in pressure. This suggestion is supported to some extent by the observation of an increase in average glomerular capillary diameter in the $\mathrm{D}_{\mathrm{Mr}}$ group [26]. Elevated glomerular pressure may then contribute to the increased albumin excretion. Since arteriolar structure varies greatly be- 
tween different nephrons albumin leakage may also do so. The failure to demonstrate a correlation between structural parameters and the AER in the present series does not exclude the possibility of a role for structural lesions. Further progression of the disease and increasing systemic blood pressure and proteinuria may in turn contribute to further basement membrane accumulation thus starting a vicious cycle.

Although diabetic patients without hypertension, by conventional WHO definition, were chosen, the group with microalbuminuria had elevated levels of diastolic pressure, as reported by several other workers [22, 27-29]. The lack of correlation between blood pressure and the structural changes in our groups may be due to the fairly narrow range of blood pressure and does not exclude an interaction between them.

The finding of structural lesions in the glomerular architecture, and in the arterioles proximal and distal to the glomerular tuft in patients with low-level albuminuria call for consideration of preventive measures as early as possible, even before clear elevations of AER and blood pressure. It is plausible to suggest that an anti-hypertensive treatment would have protective effects on the future status of the arterioles and may thereby influence the development of nephropathy.

Acknowledgements. Kidney biopsies from four normal donors included in this study were measured by R. Østerby while she was a visiting professor in the research laboratories of M.W. Steffes and S.M.Mauer of the University of Minnesota. The skilful technical assistance of Ms. B. Saugbjerg, Ms. B. Iversen and Ms. L. Lysgaard is gratefully acknowledged. Ms. A. Dalmose is thanked for the photographic work and illustrations. The study was supported by grants from Juvenile Diabetes Foundation Int. grant \#190592, The Danish Diabetes Association, The Danish Medical Research Council, NOVO Foundation, Nordic Research Committee, Århus University Research Foundation.

\section{References}

1. Rosivall L (1990) Morphology and function of the distal part of the afferent arteriole. Kidney Int 38: S10-S15

2. Pedersen MM (1994) Early renal hyperfunction and hypertrophy in IDDM patients including comments on early intervention. In: Mogensen CE (ed) The kidney and hypertension in diabetes mellitus. Kluwer Academic Publishers, Boston, pp 297-308

3. Hostetter TH, Rennke HG, Brenner BM (1982) The case for intrarenal hypertension in the initiation and progression of diabetic and other glomerulopathies. Am J Med 72: $375-380$

4. Vora JP, Anderson S, Brenner BM (1994) Pathogenesis of diabetic glomerulopathy: The role of glomerular hemodynamic factors. In: Mogensen CE (ed) The kidney and hypertension in diabetes mellitus. Kluwer Academic Publishers, Boston, pp 223-232

5. Thomsen, AaC (1965) The kidney in diabetes mellitus. Munksgaard, Copenhagen
6. Bader R, Bader H, Grund KE, Mackensen Haen S, Christ $\mathrm{H}$, Bohle A (1980) Structure and function of the kidney in diabetic glomerulosclerosis. Correlations between morphological and functional parameters. Pathol Res Pract 167: 204-216

7. Taft JL, Billson VR, Nankervis A, Kincaid Smith P, Martin FI (1990) A clinical-histological study of individuals with diabetes mellitus and proteinuria. Diabet Med 7: 215-221

8. Harris RD, Steffes MW, Bilous RW, Sutherland DE, Mauer SM (1991) Global glomerular sclerosis and glomerular arteriolar hyalinosis in insulin dependent diabetes. Kidney Int 40: 107-114

9. Lane P, Steffes MW, Mauer SM (1991) Structural-functional relationships in type I insulin-dependent diabetes mellitus in humans. J Diabetes Complications 5: 69-71

10. Mogensen CE, Chachati A, Christensen CK et al. (1985) Microalbuminuria: an early marker of renal involvement in diabetes. Uremia Invest 9: 85-95

11. Walker JD, Close CF, Jones SL et al. (1992) Glomerular structure in type-1 (insulin-dependent) diabetic patients with normo- and microalbuminuria. Kidney Int 41 : 741-748

12. Bangstad H-J, Østerby R, Dahl-Jørgensen K et al. (1993) Early glomerulopathy is present in young, type 1 (insulindependent) diabetic patients with microalbuminuria. Diabetologia 36: 523-529

13. Bangstad H-J, Østerby R, Dahl-Jørgensen K, Berg KJ, Hartmann K, Hanssen KF (1994) Improvement of blood glucose control in IDDM patients retards the progression of morphological changes in early diabetic nephropathy. Diabetologia 37: 483-490

14. Fioretto P, Steffes MW, Mauer SM (1994) Glomerular structure in nonproteinuric IDDM patients with various levels of albuminuria. Diabetes 43: 1358-1364

15. Steffes MW, Bilous RW, Sutherland DE, Mauer SM (1992) Cell and matrix components of the glomerular mesangium in type I diabetes. Diabetes 41: 679-684

16. $\emptyset$ sterby R (1992) Glomerular structural changes in type 1 (insulin-dependent) diabetes mellitus: causes, consequences, and prevention. Diabetologia 35: 803-812

17. Cagliero E, Roth T, Roy S, Lorenzi M (1991) Characteristics and mechanisms of high-glucose-induced overexpression of basement membrane components in cultured human endothelial cells. Diabetes $40: 102-110$

18. Ayo SH, Radnik RA, Garoni JA, Glass WF, Kreisberg JI (1990) High glucose causes an increase in extracellular matrix proteins in cultured mesangial cells. Am J Pathol 136: 1339-1348

19. Haneda M, Kikkawa R, Horide N et al. (1991) Glucose enhances type IV collagen production in cultured rat glomerular mesangial cells. Diabetologia 34: 198-200

20. Danne T, Spiro MJ, Spiro RG (1993) Effect of high glucose on type IV collagen production by cultured glomerular epithelial, endothelial, and mesangial cells. Diabetes 42: 170177

21. Wakisaka M, Spiro MJ, Spiro RG (1994) Synthesis of type VI collagen by cultured glomerular cells and comparison of its regulation by glucose and other factors with that of type IV collagen. Diabetes 43: 95-103

22. Wiseman M, Viberti G, Mackintosh D, Jarret RJ, Keen $H$ (1984) Glycaemia, arterial pressure and micro-albuminuria in type 1 (insulin-dependent) diabetes mellitus. Diabetologia 26: 401-405

23. Nørgaard K, Storm B, Graae M, Feldt-Rasmussen B (1988) Elevated albumin excretion and retinal changes in children with type 1 diabetes are related to long-term poor blood glucose control. Diabet Med 6: 325-328 
24. Bangstad H-J, Hanssen KF, Dahl-Jørgensen K, Aagenaes O (1989) Microalbuminuria is associated with long term poor glycemic control in adolescent insulin dependent diabetics. Diabetes Res 12: 71-74

25. The Diabetes Control and Complications Trial Research Group (1993) The effect of intensive treatment of diabetes on the development and progression of long-term complications in insulin-dependent diabetes mellitus. $\mathrm{N}$ Eng1 $\mathrm{J}$ Med 329: 977-986

26. Østerby R (1995) Microalbuminuria in diabetes mellitus is there a structural basis? Nephrol Dial Transplant 10: 1214
27. Mathiesen ER, Ronn B, Jensen T, Storm B, Deckert T (1990) Relationship between blood pressure and urinary albumin excretion in development of microalbuminuria. Diabetes 39: 245-249

28. Microalbuminuria Collaborative Study Group (1992) Microalbuminuria in type I diabetic patients. Prevalence and clinical characteristics. Diabetes Care 15: 495-501

29. Poulsen PL, Hansen KW, Mogensen CE (1994) Ambulatory blood pressure in the transition from normo- to microalbuminuria. A longitudinal study in IDDM patients. Diabetes 43: 1248-1253 